\section{Interferon-gamma release assay as a diagnostic test for tuberculosis- associated uveitis}

\author{
Abstract \\ Background To study the use of interferon- \\ gamma release assay (IFN- $\gamma$ ) (IGRAs) as a \\ diagnostic test for tuberculosis (TB)-associated \\ uveitis (TAU). \\ Design Prospective cohort study. \\ Participants Consecutive new patients \\ ( $n=162)$ with clinical ocular signs suggestive \\ of TAU, seen $>1$ year period at a single \\ tertiary center. \\ Methods All subjects underwent
}

${ }^{1}$ Singapore National Eye Centre, National University of Singapore, Singapore, Singapore

${ }^{2}$ Singapore Eye Research Institute, National University of Singapore, Singapore, Singapore

${ }^{3}$ Singapore Genera Hospital, Pathology, National University of Singapore, Singapore, Singapore

${ }^{4}$ Department of Ophthalmology, Yong Yoo Lin School of Medicine, National University of Singapore, Singapore, Singapore

Correspondence: S-P Chee, Singapore National Eye Centre, 11 Third Hospital Avenue, Singapore 168751, Singapore

Tel: + 6562277255

Fax: + 6562277290 .

E-mail: chee.soon.phaik@

snec.com.sg

Received: 21 February 2011 Accepted in revised form: 29 November 2011

Published online:

3 February 2012 investigations to rule out underlying disease, including T-SPOT.TB and tuberculin skin test (TST). Twenty-one subjects with underlying disease and three with interdeterminate T-SPOT.TB results were excluded. Those with T-SPOT.TB- or TST-positive results were referred to infectious diseases physician for evaluation. Anti-TB therapy (ATT) was prescribed if required. Patients' treatment response and recurrence were monitored for six months after completion of ATT, if given; or 1 year if no ATT was given.

Main outcome measure Diagnosis of TAU.

Results Mean age of study cohort $(n=138)$ was $46.8 \pm 15.3$ years. Majority were Chinese $(n=80,58.0 \%)$ and female $(n=75,54.3 \%)$. TST was more sensitive than T-SPOT.TB $(72.0 \%$ vs $36.0 \%$ ); but T-SPOT.TB was more specific (75.0\% vs $\mathbf{5 1 . 1 \%}$ ) for diagnosing TAU. Patients with either a T-SPOT.TB $(1.44 ; 95 \%$ confidence intervals (CI), $0.86-2.42)$ or TST $(1.47 ; 95 \% \mathrm{CI}$, 1.12-1.94)-positive result are more likely to have TAU. The accuracy of diagnosing TAU increases when both tests are used in combination (area under the receiver operator curve $=0.665 ; 95 \%$ CI, 0.533-0.795). Patients with both tests positive are 2.16 (95\% CI, 1.23-3.80) times more likely to have TAU. Negative T-SPOT.TB or TST results do not exclude TAU (negative likelihood ratios $<1.0$ ).
M Ang ${ }^{1,2}$, W Wong ${ }^{2}, C C L ~ N g a n^{3}$ and S-P Chee ${ }^{1,4}$

Conclusions We recommend using a combination of clinical signs, IGRA, and TST to diagnose TAU.

Eye (2012) 26, 658-665; doi:10.1038/eye.2012.1; published online 3 February 2012

Keywords: tuberculosis; uveitis; interferongamma release assay

Introduction

The WHO (World Health Organization) in 2009 reported an increasing number of tuberculosis (TB) infections in both the developing and developed world due to multi-drug resistant TB, HIV, and global migration. ${ }^{1-3}$ It is estimated that $70-80 \%$ of all TB infections in immunocompetent individuals are latent. ${ }^{1}$

Mycobacterium tuberculosis (MTB) can infect the eye directly or cause inflammation by antigenic mimicry, whereby the host produces a cross-reactive immune response to an unseen source of TB infection. ${ }^{4-6}$ Unfortunately, tests such as of MTB cultures, acid-fast bacilli (AFB) smear, or PCR detection of MTB DNA from ocular samples have low sensitivities (20-30\%). Both the immune-mediated mechanism of inflammation as well as small inoculum of MTB in the eye may account for this.

The incidence of TB in Singapore is about 40 per 100000 per year, compared with Western Europe and the US ( $<25$ per 100000) and high burden countries such as India ( $>300$ per 100 000) - (Global tuberculosis control: a short update to the 2009 report $\mathrm{WHO} / \mathrm{HTM} / \mathrm{TB} /$ 2009.426). Currently, the diagnosis and treatment of TB still depend on the century-old Mantoux test or tuberculin skin test (TST) which is still routinely performed on all patients with uveitis in our clinical practice. ${ }^{7}$ TST has a low specificity due false-positive response in 
persons infected with non-tuberculous mycobacterium or vaccinated with BCG. ${ }^{8,9}$ IGRAs such as T-SPOT.TB (Oxford Immunotec, Oxford, UK) and QuantiFERON-TB Gold In-tube or QFT (Cellestis Incorporated, Carnegie, Australia) are more specific and sensitive than TST in detecting active pulmonary TB infections. ${ }^{10}$ However, they are less sensitive for diagnosing latent TB infections (LTBI). ${ }^{11}$ In Singapore, T-SPOT.TB was found to be more sensitive than QFT when both tests were evaluated for diagnosing pulmonary TB. ${ }^{12,13}$ However, T-SPOT.TB has not been studied specifically for diagnosing tuberculosisassociated uveitis (TAU). In this study, we compared T-SPOT.TB with the TST as a diagnostic test for TAU.

\section{Materials and methods}

\section{Study participants and overview of management}

We conducted a prospective cohort study of all new consecutive patients with uveitis presenting to the Singapore National Eye Centre (SNEC) Ocular Inflammation and Immunology Service $>1$ year period (1 September 2008 to 31 August 2009). Ethics approval was obtained from our local Institutional Review Board. Patients were enrolled if they had clinical ocular signs suspicious of TAU such as granulomatous inflammation, broad-based posterior synechiae, retinal vasculitis with or without choroiditis, and serpiginous-like choroiditis, as defined by Tabbara ${ }^{21,41}$ and Gupta et al, ${ }^{4,14}$ and consented to participate in the study. All applicable institutional and governmental regulations concerning the ethical use of human volunteers/animals were followed during this research.

Briefly, all study subjects underwent a full systemic review, ocular examination, and standard baseline investigations - Figure 1. Blood was taken for T-SPOT.TB before TST was performed. Patients were excluded if they had (1) any other possible infectious or non-infectious cause to account for the uveitis or (2) a T-SPOT.TB result that was indeterminate as these tests cannot be interpreted. All patients were referred to the infectious diseases physician at Singapore General Hospital for review and were prescribed Anti-TB therapy (ATT) if required. Patients were followed up every 2 weeks for 8 weeks, then 2-3 monthly (or more frequently as required) to monitor response to therapy. Systemic corticosteroids were added if there was any increase in ocular inflammation after commencing $\mathrm{ATT}^{15}$ defined as a two-step increase in inflammation using the Standardization of Uveitis Nomenclature (SUN) working group activity score. ${ }^{16}$ Patients were followed up for a period of 6 months after completion of therapy (if ATT was given) or a minmum of 1 year if no ATT was given (whichever was longer).

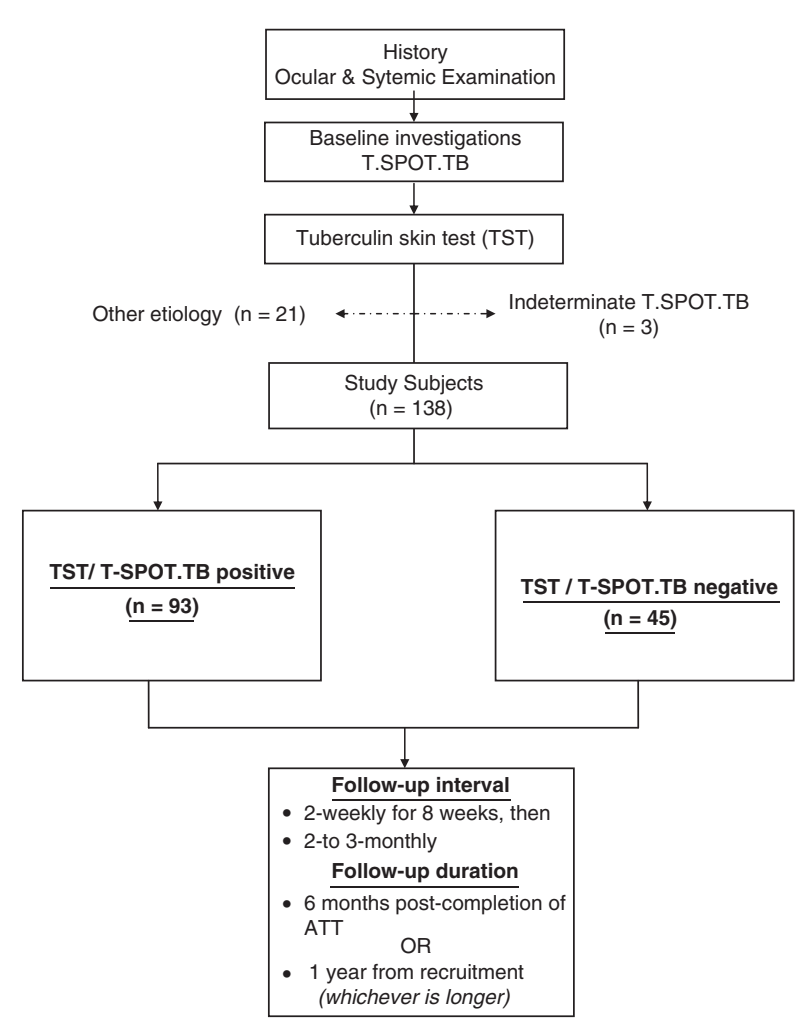

Figure 1 Flowchart depicting study cohort and follow-up. $n$, number of study subjects; ATT, anti-tubercular treatment.

\section{Investigations}

All patients had standard baseline investigations such as complete blood count, erythrocyte sedimentation rate, liver enzyme panel, infectious disease screen (which included Venereal Disease Research Laboratory (VDRL) test for syphilis, TST, urine microscopy), and a chest $X$ ray (CXR). Other tests such as QFT, AFB smears from throat swabs or PCR assays for TB DNA were performed in patients with severe anterior chamber inflammation to exclude TB.

T-SPOT.TB was performed according to the manufacturer's instructions, and blood was taken before the TST was administered. ${ }^{17}$ For each patient, $8 \mathrm{ml}$ of blood was collected in Lithium Heparin tubes and processed within $8 \mathrm{~h}$. Peripheral blood mononuclear cells (PBMCs) were prepared by density gradient centrifugation over Ficoll PaquePlus (GE Healthcare Bio-Sciences AB, Uppsala, Sweden). A total of 250000 cells were seeded in each of four wells of the assay plate. The cells were stimulated for 16-20 $\mathrm{h}$ (under $5 \%$ carbon dioxide at $37^{\circ} \mathrm{C}$ ) with medium, GIBCO AIM-V (Invitrogen Corporation, Carlsbad, CA, USA) (nil control), phytohaemagglutinin (mitogen-positive control), or the TB-specific peptide antigens (peptide pools for early secretory antigenic target-6 (ESAT-6) (Panel A) and for culture filtrate protein-10 (CFP-10) (Panel B), in separate wells) in a total volume of $150 \mu \mathrm{l}$ 
per well. Two readers quantified the number of IFN- $\gamma$ spot-forming $\mathrm{T}$ cells visually, and a third reader was consulted if results were disparate.

TST was performed by using the standard Mantoux method: intradermal injection of $0.1 \mathrm{ml}$ (two tuberculin units (T.U.)) purified protein derivative (RT23 SSI 2 T.U./0.1 ml Statens Serum Institut, Copenhagen, Denmark). Induration was measured at 72 hours with a ruler and considered positive if it measured $\geq 15 \mathrm{~mm}$ (as validated in our population). ${ }^{9,18}$ A CXR with evidence of pulmonary nodules, with or without visible calcification and/or fibrotic scars in the hilar area or upper lobes was considered as a positive CXR finding. ${ }^{7}$

\section{Treatment and management of patients}

The infectious diseases physician at the Singapore General Hospital independently evaluated all patients with a high clinical index of suspicion for $\mathrm{TB}$, positive TST or T-SPOT.TB. Those found to have associated systemic or pulmonary TB infection received ATT, whereas uveitis patients with LTBI were advised on the risk-benefit ratio of ATT. ${ }^{19}$ Patients consenting to treatment received standard ATT according to CDC guidelines (isoniazid $5 \mathrm{mg} / \mathrm{kg}$ daily, rifampicin 450-600 mg daily, pyrazinamide $30 \mathrm{mg} / \mathrm{kg}$ daily, and ethambutol $15 \mathrm{mg} / \mathrm{kg}$ daily for 2 months, followed by two drugs for a 4-month continuation phase, for a total minimum of 6 months duration). ${ }^{19,20}$

In patients with posterior segment inflammation where ATT was not indicated, oral prednisolone was used at a starting dosage of $1 \mathrm{mg} / \mathrm{kg}$ body weight, tapering slowly over the clinical course was given. Any anterior segment inflammation was treated with topical corticosteroids. The therapeutic response was monitored by one ophthalmologist (SPC), where a two-step decrease in inflammation (SUN working group activity score) was considered an improvement in clinical activity and a positive response to treatment. ${ }^{16}$

\section{Defintion of TAU}

The final diagnosis of TAU was only made at the end of our study, after all patients had completed treatment and follow-up. We adapted our criteria from published definitions by Gupta et $a l^{4}$ and Tabbara. ${ }^{21}$ A definite diagnosis of ocular TB infection was made in patients with a positive AFB smear, MTB culture, or PCR assay from ocular samples. ${ }^{21}$ Patients without an underlying disease who responded to corticosteroid therapy alone and no ATT, with no recurrence within 1 year were presumed not to have TAU. Patients with no definite source of TB infection, who responded to ATT within 4-6 weeks of treatment without recurrence for 6 months following completion of therapy, having had other diseases excluded were presumed to have TAU. ${ }^{4}$

\section{Statistical analysis}

We studied the final diagnoses of all patients and compared their TST or T-SPOT.TB results after 6 months of completing therapy (Figure 2). We classified each patient's anatomical location, course, and laterality of ocular inflammation according to the International Uveitis Study Group clinical classification criteria. ${ }^{22}$ Statistical analysis included descriptive statistics, where the mean and SD with 95\% CI was calculated for the continuous variables whereas frequency distribution and

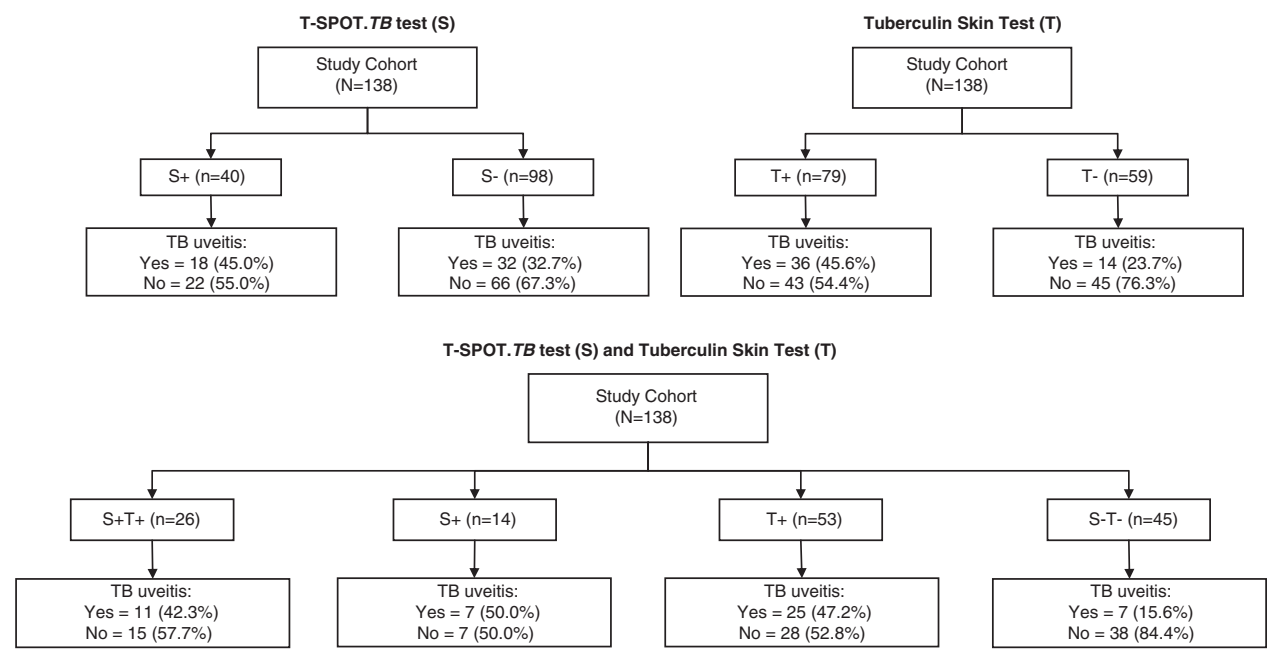

Figure 2 Final diagnoses of study cohort following T-SPOT.TB test (S), tuberculin skin test $(\mathrm{T})$ and when used in combination $(\mathrm{S}+\mathrm{T})$. + , positive; - , negative. 
percentages were used for categorical variables. One-way ANOVA was used to compare means of outcome groups for each characteristic variable whereas Pearson's square $\chi^{2}$-tests (incorporating Yates' correction if necessary) were used to assess the independent association between characteristic variables and outcome groups. A $P$-value $<0.05$ was considered statistically significant.

Performances of binary classification tests were evaluated using sensitivity, specificity, and area under the receiver operator curve (AUC). All analyses were performed using STATA version 11 (StataCorp LP, College Station, TX, USA).

\section{Results}

A total of 162 consecutive, new patients with uveitis were enrolled during our study period (Figure 1). After initial assessment, we excluded 21 patients (13.0\%) with underlying diseases that confound the final diagnosis of TAU and three patients (1.9\%) with indeterminate T-SPOT.TB results (Figure 1). Patients excluded were HLA-B27 positive $(n=10)$, VDRL positive $(n=5)$, or those diagnosed with herpetic anterior uveitis $(n=4)$ and sarcoidosis $(n=2)$. All three patients with indeterminate T-SPOT.TB results had unilateral acute anterior uveitis successfully treated with topical steroids, with no recurrence of inflammation at the end of our study period. All remaining 138 patients did not have an infectious or non-infectious cause for ocular inflammation detected by the end of their follow-up period.
The clinical characteristics of the 138 study subjects are seen in Table 1 . The mean age of these patients was $46.8 \pm 15.3$ years. The majority were Chinese $(n=80$, $58.0 \%)$ and female $(n=75,54.3 \%)$. We found no significant differences in terms of age, gender, race, or anatomical classification of uveitis between patients with positive or negative T-SPOT.TB results. Patients presented with ocular signs consistent with a tubercular cause such as granulomatous $(n=10)$ or medium keratic precipitates $(n=52)$; iris nodules $(n=16)$; broad and extensive posterior synechiae $(n=36)$; choroiditis $(n=14)$, serpinginous choroiditis, $(n=1)$ or vasculitis $(n=33)$. There were no cases of definite ocular TB infection in this study cohort as nine patients had vitreous biopsy and were found to have no evidence of a positive AFB smear, MTB culture, or PCR assay from these ocular samples. However, five patients (3.7\%) had AFB smear-positive sputum samples and two (1.5\%) patients had positive PCR results from urine samples. The majority of patients $(124 / 138,89.9 \%)$ had CXR findings that were not suggestive of pulmonary TB infection, as defined. ${ }^{7}$ A total of 50 patients were presumed TAU as defined in our study, and completed ATT for a median duration of 6.9 (range 6-9) months. All patients who were treated with ATT resolved with no recurrence. The remaining patients $(n=88)$ were presumed negative and were responsive to corticosteroid therapy only.

The study cohort was divided into four main groups, as illustrated in Figure 2: $\mathrm{S}+\mathrm{T}+$ (both T-SPOT.TB and TST positive), $\mathrm{T}+$ (only TST positive, T-SPOT.TB negative), $\mathrm{S}+$ (only T-SPOT.TB positive, TST negative),

Table 1 Demographics and anatomical classification of uveitis in study cohort

\begin{tabular}{|c|c|c|c|c|}
\hline \multirow[t]{2}{*}{ Characteristics } & \multirow[t]{2}{*}{ All $(\mathrm{n}=138)$} & \multicolumn{3}{|c|}{ T-SPOT.TB result } \\
\hline & & Positive $(\mathrm{n}=40)$ & Negative $(\mathrm{n}=98)$ & P-value ${ }^{\mathrm{a}}$ \\
\hline Age, years $( \pm S D)$ & $46.8(15.3)$ & $46.9(14.4)$ & $47.7(15.5)$ & 0.74 \\
\hline \multicolumn{5}{|l|}{ Gender (\%) } \\
\hline Male & $63(45.7)$ & $23(57.5)$ & $28(40.8)$ & \multirow{2}{*}{0.09} \\
\hline Female & $75(54.3)$ & $17(42.5)$ & $29(59.2)$ & \\
\hline \multicolumn{5}{|l|}{ Race (\%) } \\
\hline Chinese & $80(58.0)$ & $21(52.5)$ & $59(60.2)$ & \multirow{5}{*}{0.09} \\
\hline Malay & $12(8.7)$ & $1(2.5)$ & $11(11.2)$ & \\
\hline Indian & $27(19.6)$ & $13(32.5)$ & $14(14.3)$ & \\
\hline Caucasian & $2(1.4)$ & $0(0.0)$ & $2(2.0)$ & \\
\hline Others & $17(12.3)$ & $5(12.5)$ & $12(12.3)$ & \\
\hline \multicolumn{5}{|l|}{ Type of Uveitis ${ }^{b}(\%)$} \\
\hline Anterior & $66(48.2)$ & $21(52.5)$ & $45(46.4)$ & \multirow{4}{*}{0.34} \\
\hline Intermediate & $14(10.2)$ & $1(2.5)$ & $13(13.4)$ & \\
\hline Posterior & 31 (22.6) & 11 (27.5) & $20(20.6)$ & \\
\hline Panuveitis & $26(19.0)$ & 7 (17.5) & $19(19.6)$ & \\
\hline
\end{tabular}

${ }^{\text {a }}$-value from one-way ANOVA or $\chi^{2}$-test as appropriate.

${ }^{\mathrm{b}}$ Anatomical classification according to the Standardized Uveitis Nomenclature (SUN) Working group. 
Table 2 Comparison of accuracy between T-SPOT.TB (S), Tuberculin skin test (T), and combination (S + T)

\begin{tabular}{lcrr}
\hline Features of diagnostic tests & \multicolumn{3}{c}{ Diagnostic tests, (95\%CI) } \\
\cline { 2 - 4 } & \multicolumn{1}{c}{$S$} & $T$ & $S+T$ \\
\hline Sensitivity & $36.0 \%(24.1,49.9)$ & $72.0 \%(58.3,82.5)$ & $61.1 \%(38.6,79.7)$ \\
Specificity & $75.0 \%(65.0,82.9)$ & $51.1 \%(40.9,61.3)$ & $71.7(58.4,82.0)$ \\
Positive predictive value & $45.0 \%(30.7,60.2)$ & $45.6 \%(35.1,56.5)$ & $42.3 \%(25.5,61.1)$ \\
Negative predictive value & $67.4 \%(57.6,75.8)$ & $76.3 \%(64.0,85.3)$ & $84.4 \%(71.2,92.3)$ \\
Positive likelihood ratio & $1.44(0.86,2.42)$ & $1.47(1.12,1.94)$ & $2.16(1.23,3.80)$ \\
Negative likelihood ratio & $0.85(0.67,1.09)$ & $0.54(0.34,0.89)$ & $0.54(0.30,0.99)$ \\
Accuracy & $60.9 \%(52.7,69.0)$ & $58.7 \%(50.5,66.9)$ & $69.0 \%(57.5,78.6)$ \\
AUC & $0.555(0.474,0.636)$ & $0.616(0.534,0.698)$ & $0.665(0.533,0.795)$
\end{tabular}

Abbreviations: CI, confidence intervals; AUC, area under the receiver operator characteristic curve; S, T-SPOT.TB; T, Tuberculin skin test.

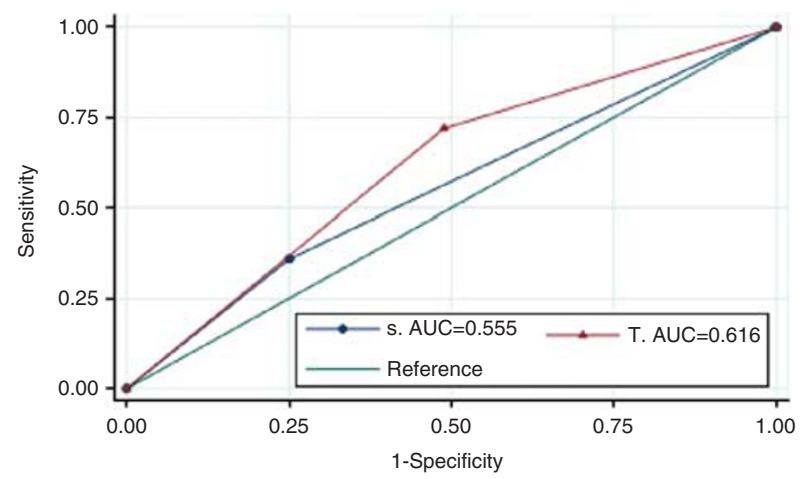

Figure 3 AUC for tuberculin skin test (T) and T-SPOT.TB (S).

and S-T- (neither test positive). The statistical basis of our study involves the comparison of these four groups and their respective final diagnosis of TAU. We estimated the sensitivities, specificities, and AUC of each test individually and when used in combination (Table 2). In the analysis of each test individually, the TST (60.9\%; 95\% CI, 52.7-69.0) has a higher diagnostic accuracy than the T-SPOT.TB (58.7\%; 95\% CI, 50.5-66.9). Although the TST is more sensitive as compared with T-SPOT.TB $(72.0 \%$ vs $36.0 \%)$, the latter is more specific for diagnosis (75.0 vs 51.1\%). The incorporated ROC (AUC) value for TST $(0.616 ; 95 \%$ CI, 0.534-0.698) was greater than for T-SPOT.TB (0.555; 95\% CI, 0.474-0.636) - Figure 3.

When both T-SPOT.TB and TST are used in combination, the overall accuracy for predicting TAU increases to $69.0 \%(95 \% \mathrm{CI}, 57.5-78.6 \%)$ with a higher AUC of 0.665 (95\% CI, 0.533-0.795) - Figure 4. If both tests are positive $(\mathrm{S}+\mathrm{T}+)$, the positive likelihood ratio is 2.16 (95\% CI, 1.23-3.80). In patients with both tests negative, $82.2 \%(37 / 45)$ patients resolved with no recurrence despite treatment without ATT. However, the negative likelihood ratio was $<1.0$ for patients with both tests negative $(0.54 ; 95 \% \mathrm{CI}, 0.30-0.99)$. The concordance of both tests was low $(\kappa$-value $=0.085(P=0.239))$ in our

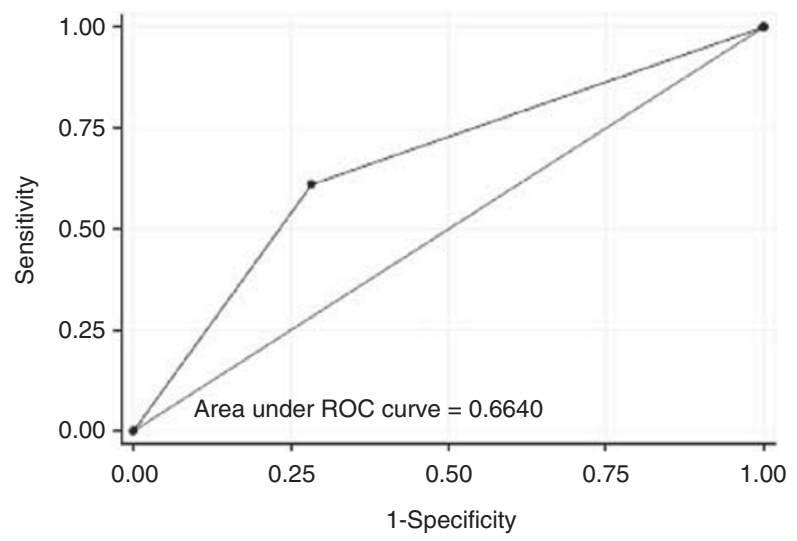

Figure 4 AUC combining tuberculin skin test $(\mathrm{T})$ and T-SPOT.TB (S).

study cohort. Discordant results were found in 67/138 $(48.6 \%)$ cases. In these patients, the positive likelihood ratios were comparable in patients with $\mathrm{S}+\mathrm{T}-(1.44$; 95\% CI, 0.86-2.42) and S-T + (1.47; 95\% CI, 1.12-1.94); whereas the negative likelihood ratios were $<1.0$ for patients with $\mathrm{S}+\mathrm{T}-(0.85 ; 95 \% \mathrm{CI}, 0.67-1.09)$ and $\mathrm{S}-\mathrm{T}+$ (0.54; 95\% CI, 0.34-0.89). No significant risk factors were identified for discordant results such as age $(P=0.38)$, gender $(P=0.73)$, race $(P=0.33)$, or type of uveitis $(P=0.54)$. We used the observed prevalence of TAU to compare the prior probability of disease with the positive and negative predictive values (PPV and NPV, respectively). Although T-SPOT.TB and TST each had a greater PPV $(45.0 \%$ and $45.6 \%$, respectively $v s$ the prior probability of $36.2 \%$ - an improvement of $9 \%$ ), combining the test results improved the PPV over the prior probability by $17 \%$.

\section{Discussion}

Our study found that patients with suggestive clinical signs of TB plus either a positive TST or T-SPOT.TB were 
approximately 1.5 times more likely to have TAU as compared with patients with negative tests. We also found that T-SPOT.TB is more specific but less sensitive than TST, and when both tests are used in combination the overall accuracy for diagnosing TAU increases. Patients with suggestive clinical signs in addition to positive TST and T-SPOT.TB have a two times increased likelihood of having TAU. On the other hand, we found that both tests (used individually or in combination) have poor sensitivities. Therefore, neither a negative T-SPOT.TB nor a negative TST result, in patients who have ocular signs suggestive of a tubercular cause, adequately excludes TAU (negative likelihood ratios $<1.0$ for both tests individually and in combination - Table 2).

In our clinical practice, a high index of suspicion is required to diagnose TAU. Current clinical practice involves diagnosing TAU by using a positive TST and suggestive clinical signs such as broad-based posterior synechiae, retinal vasculitis with or without choroiditis, and serpiginous-like choroiditis. ${ }^{14}$ Our study found that adding T-SPOT.TB increases the discrimination and accuracy of diagnosing TAU. However, it must be noted that the AUC value of combining T-SPOT.TB and TST with clinical signs is only fairly good for discrimination (AUC $=0.665$ ). Our findings are consistent with published studies, which found low sensitivities for T-SPOT.TB and TST in patients with extrapulmonary and latent TB. ${ }^{23}$ This makes negative T-SPOT.TB or TST results difficult to interpret, as up to $20 \%$ of persons with negative tests may still have TB infection. ${ }^{24,25}$ However, the results of our study should be taken in consideration together with the prevalence of disease and the prior probability of disease. In the same study period, we diagnosed TAU in 50 out of 621 patients with uveitis $(8 \%)$, which is consistent with our intermediate burden of TB disease. The estimated prevalence of tubercular uveitis range from 1-4\% areas with low TB endemicity such as USA, Europe, and Japan to 10-26\% in highly endemic regions such as India and Saudi Arabia. $^{26-28}$

T-SPOT.TB is an objective, reproducible blood test that requires only one visit to detect TB infection. ${ }^{11}$ It is more specific as it use two MTB proteins, ESAT-6, and CFP-10 to stimulate IFN- $\gamma$ response from circulating CD4 T-cells. ${ }^{17}$ The main disadvantage of T-SPOT.TB is its higher cost and the need for trained personnel. Moreover, the handling and transport of blood samples T-SPOT.TB is both time and temperature sensitive. Thus, for it to be cost-effective, T-SPOT.TB was recommended only in those with a positive TST result. ${ }^{29}$ Our study found that the agreement between the TST and T-SPOT.TB to be low but unlike other studies did not find any significant contributory factors for discordant results such as age, gender, or race. ${ }^{30,31}$
We recommend that both tests be performed simultaneously and interpreted with clinical signs, as T-SPOT.TB complements TST to improve the accuracy of diagnosing TAU. This is consistent with our previous recommendation of using QFT and TST. ${ }^{32}$ T-SPOT.TB differs from QFT in that the former involves harvesting and counting viable PMBCs that release IFN- $\gamma$ whereas the latter uses an enzyme-linked immunosorbent assay to study IFN- $\gamma$ release from T-cells in whole blood. The technique used in T-SPOT.TB may have better resolution of blood samples with reduced T-cell numbers (for example, samples from immunocompromised individuals) that would usually give indeterminate QFT results. ${ }^{11}$

Recent efforts by WHO to stop the spread of TB underline the need for early and accurate diagnosis of TB. $^{33}$ TAU is often diagnosed late and under-treated. ${ }^{34}$ ATT has been shown to reduce recurrence in patients with TAU. ${ }^{35}$ However, owing to the long course of multiple medications and their potential side effects, clinicians and patients alike are averse to ATT. TST is highly subjective, non-specific for MTB, and may be affected by the patient's immune system. ${ }^{36}$ This reinforces our recommendation to perform both T-SPOT.TB and TST to guide treatment decisions.

The main limitation of all studies involving extrapulmonary TB including TAU is the limited number of patients who have a positive TB culture, which is the gold standard for diagnosis. ${ }^{11,32,37,38}$ We used a recognized definition of TAU in our study that was used in many other studies and indeed, in our clinical practice. ${ }^{4,21,32,34,35,39-41}$ Our study is also limited by the relatively small sample size, but to our knowledge, is the largest prospective study on T-SPOT.TB used specifically for the diagnosis of TAU. Currently, logistical and cost-related issues restrict the use of T-SPOT.TB in our daily clinical practice.

In conclusion, we found that although T-SPOT.TB is more specific for TAU, it serves as a better diagnostic tool if used in conjunction with the TST. Therefore, we recommend that T-SPOT.TB be used as an adjunctive tool to be used with clinical signs and TST in the diagnosis of TAU.

Summary

What was known before

- T-SPOT.TB is a useful diagnostic test for pulmonary tuberculosis infections. T-SPOT.TB may not be as useful for diagnosing tuberculosis-associated uveitis.

What this study adds

- T-SPOT.TB is a useful adjunct to diagnosing tuberculosisassociated uveitis. T-SPOT.TB adds discrimination (using area under the receiver operator curve) when used together with clinical signs, suggestive of tuberculosis and Mantoux. 


\section{Conflict of interest}

The authors declare no conflict of interest.

\section{Acknowledgements}

All authors had full access to all the data in the study and take responsibility for the integrity of the data and the accuracy of the data analysis.

\section{References}

1 WHO. Global tuberculosis control: key findings from the December 2009 WHO report. Wkly Epidemiol Rec 2010; 85(9): 69-80.

2 Blumberg HM, Migliori GB, Ponomarenko O, Heldal E. Tuberculosis on the move. Lancet 2010; 375(9732): 2127-2129.

3 Gandhi NR, Nunn P, Dheda K, Schaaf HS, Zignol M, van Soolingen D et al. Multidrug-resistant and extensively drugresistant tuberculosis: a threat to global control of tuberculosis. Lancet 2010; 375(9728): 1830-1843.

4 Gupta V, Gupta A, Rao NA. Intraocular tuberculosis - an update. Surv Ophthalmol 2007; 52(6): 561-587.

5 Garip A, Diedrichs-Mohring M, Thurau SR, Deeg CA, Wildner G. Uveitis in a patient treated with BacilleCalmette-Guerin: possible antigenic mimicry of mycobacterial and retinal antigens. Ophthalmology 2009; 116(12): 2457-2462 e2451-2452.

6 Spratt A, Key T, Vivian AJ. Chronic anterior uveitis following bacille Calmette-Guerin vaccination: molecular mimicry in action? J Pediatr Ophthalmol Strabismus 2008; 45(4): 252-253.

7 American Thoracic Society. Targeted tuberculin testing and treatment of latent tuberculosis infection. MMWR Recomm Rep 2000; 49(RR-6): 1-51.

8 Morimura Y, Okada AA, Kawahara S, Miyamoto Y, Kawai S, Hirakata A et al. Tuberculin skin testing in uveitis patients and treatment of presumed intraocular tuberculosis in Japan. Ophthalmology 2002; 109(5): 851-857.

9 Chee $\mathrm{CB}$, Soh $\mathrm{CH}$, Boudville IC, Chor SS, Wang YT. Interpretation of the tuberculin skin test in Mycobacterium bovis BCG-vaccinated Singaporean schoolchildren. Am J Respir Crit Care Med 2001; 164(6): 958-961.

10 Leung CC, Yam WC, Yew WW, Ho PL, Tam CM, Law WS et al. T-Spot.TB outperforms tuberculin skin test in predicting tuberculosis disease. Am J Respir Crit Care Med 2010; 182(6): 834-840

11 Kleinert S, Kurzai O, Elias J, Marten K, Engelke C, Feuchtenberger $\mathrm{M}$ et al. Comparison of two interferongamma release assays and tuberculin skin test for detecting latent tuberculosis in patients with immune-mediated inflammatory diseases. Ann Rheum Dis 2010; 69(4): 782-784.

12 Chee CB, Gan SH, Khinmar KW, Barkham TM, Koh CK, Liang $\mathrm{S}$ et al. Comparison of sensitivities of two commercial gamma interferon release assays for pulmonary tuberculosis. J Clin Microbiol 2008; 46(6): 1935-1940.

13 Chee CB, Lim LK, Barkham TM, Koh DR, Lam SO, Shen L et al. Use of a $\mathrm{T}$ cell interferon-gamma release assay to evaluate tuberculosis risk in newly qualified physicians in Singapore healthcare institutions. Infect Control Hosp Epidemiol 2009; 30(9): 870-875.
14 Gupta A, Bansal R, Gupta V, Sharma A, Bambery P. Ocular signs predictive of tubercular uveitis. Am J Ophthalmol 2010; 149(4): 562-570.

15 Cheung CM, Chee SP. Jarisch-Herxheimer reaction: paradoxical worsening of tuberculosis chorioretinitis following initiation of antituberculous therapy. Eye (Lond) 2009; 23(6): 1472-1473.

16 Jabs DA, Nussenblatt RB, Rosenbaum JT. Standardization of uveitis nomenclature for reporting clinical data. Results of the First International Workshop. Am J Ophthalmol 2005; 140(3): 509-516.

17 Wagstaff AJ, Zellweger JP. T-SPOT. TB: an in vitro diagnostic assay measuring T-cell reaction to Mycobacterium tuberculosis-specific antigens. Mol Diagn Ther 2006; 10(1): 57-63; discussion 64-55.

18 Cutter J, Wang YT. Tuberculosis - an under-appreciated disease. Ann Acad Med Singapore 2010; 39(3): 261-262.

19 Chee CB, Teleman MD, Boudville IC, Do SE, Wang YT. Treatment of latent TB infection for close contacts as a complementary TB control strategy in Singapore. Int J Tuberc Lung Dis 2004; 8(2): 226-231.

20 MMWR. Treatment of tuberculosis. MMWR Recomm Rep 2003; 52(RR-11): 1-77.

21 Tabbara KF. Tuberculosis. Curr Opin Ophthalmol 2007; 18(6): 493-501.

22 Deschenes J, Murray PI, Rao NA, Nussenblatt RB. International Uveitis Study Group (IUSG): clinical classification of uveitis. Ocul Immunol Inflamm 2008; 16(1): $1-2$.

23 Menzies D, Pai M, Comstock G. Meta-analysis: new tests for the diagnosis of latent tuberculosis infection: areas of uncertainty and recommendations for research. Ann Intern Med 2007; 146(5): 340-354.

24 Steiner P, Rao M, Victoria MS, Jabbar H, Steiner M. Persistently negative tuberculin reactions: their presence among children with culture positive for Mycobacterium tuberculosis (tuberculin-negative tuberculosis). Am J Dis Child 1980; 134(8): 747-750.

25 Pottumarthy S, Morris AJ, Harrison AC, Wells VC. Evaluation of the tuberculin gamma interferon assay: potential to replace the Mantoux skin test. J Clin Microbiol 1999; 37(10): 3229-3232.

26 Abu El-Asrar AM, Abouammoh M, Al-Mezaine HS. Tuberculous uveitis. Int Ophthalmol Clin 2010; 50(2): 19-39.

27 Rathinam SR, Cunningham Jr ET. Infectious causes of uveitis in the developing world. Int Ophthalmol Clin 2000; 40(2): 137-152.

28 Chang JH, Wakefield D. Uveitis: a global perspective. Ocul Immunol Inflamm 2002; 10(4): 263-279.

29 Diel R, Wrighton-Smith P, Zellweger JP. Cost-effectiveness of interferon-gamma release assay testing for the treatment of latent tuberculosis. Eur Respir J 2007; 30(2): 321-332.

30 Altet-Gomez N, De Souza-Galvao M, Latorre I, Milà C, Jiménez MA, Solsona J et al. Diagnosing TB infection in children: analysis of discordances using in vitro tests and tuberculin skin test. Eur Respir J 2011; 37(5): 1166-1174.

31 Nienhaus A, Schablon A, Diel R. Interferon-gamma release assay for the diagnosis of latent TB infection - analysis of discordant results, when compared to the tuberculin skin test. PLoS One 2008; 3(7): e2665.

32 Ang M, Htoon HM, Chee SP. Diagnosis of tuberculous uveitis: clinical application of an interferon-gamma release assay. Ophthalmology 2009; 116(7): 1391-1396. 
33 Vashishtha VM. WHO Global Tuberculosis Control Report 2009: tuberculosis elimination is a distant dream. Indian Pediatr 2009; 46(5): 401-402.

34 Cimino L, Herbort CP, Aldigeri R, Salvarani C, Boiardi L. Tuberculous uveitis, a resurgent and underdiagnosed disease. Int Ophthalmol 2009; 29(2): 67-74.

35 Bansal R, Gupta A, Gupta V, Dogra MR, Bambery P, Arora SK. Role of anti-tubercular therapy in uveitis with latent/ manifest tuberculosis. Am J Ophthalmol 2008; 146(5): 772-779.

36 Pai M, Kalantri S, Menzies D. Discordance between tuberculin skin test and interferon-gamma assays. Int J Tuberc Lung Dis 2006; 10(8): 942-943.

37 Sadatsafavi M, Shahidi N, Marra F, FitzGerald MJ, Elwood KR, Guo N et al. A statistical method was used for the metaanalysis of tests for latent TB in the absence of a gold standard, combining random-effect and latent-class methods to estimate test accuracy. J Clin Epidemiol 2010; 63(3): 257-269.
38 Vasconcelos-Santos DV, Zierhut M, Rao NA. Strengths and weaknesses of diagnostic tools for tuberculous uveitis. Ocul Immunol Inflamm 2009; 17(5): 351-355.

39 Alvarez GG, Roth VR, Hodge W. Ocular tuberculosis: diagnostic and treatment challenges. Int J Infect Dis 2009; 13(4): 432-435.

40 Babu K, Satish V, Satish S, Subbakrishna DK, Abraham MP, Murthy KR. Utility of QuantiFERON TB gold test in a south Indian patient population of ocular inflammation. Indian J Ophthalmol 2009; 57(6): 427-430.

41 Hamade IH, Tabbara KF. Complications of presumed ocular tuberculosis. Acta Ophthalmol 2010; 88(8): 905-909.

(c) This work is licensed under the Creative SOWERICHIS RESERVIED Commons Attribution-NonCommercial-No Derivative Works 3.0 Unported License. To view a copy of this license, visit http://creativecommons.org/licenses/ by-nc-nd/3.0/ 